\title{
Slight but Persistent Haematuria
}

It is assumed that the patient has been cystoscoped and that the cause of the persistent haematuria is known. The problem may arise with, for example, post-radiotherapy telangiectasia, carcinoma of prostate or low-grade widespread bladder tumours. It is assumed also that further cystoscopy is not in the interest of the patient and that conservative means should be employed.

1. You must ensure that the urine is not infected.

2. Check clotting indices such as PT, APTT, platelet count, etc.

3. If clotting factors are abnormal, intravenous vitamin $K$, an infusion of platelets or fresh frozen plasma may be of value.

4. Catheterisation and irrigation should be used only if clotting is a problem, but be aware that the very presence of the catheter may in some cases be the cause of persistent slight haematuria.

5. Try Cyklokapron (tranexamic acid) $1.5 \mathrm{~g}$ t.d.s. orally. This is an antifibrinolytic agent which can also be given intravenously. Cyklokapron should be used for a maximum of 5 days only.

6. If Cyklokapron fails, try Dicynene IG 6-hourly orally. Dicynene acts by maintaining small-vessel integrity but has no direct action on the clotting mechanism. Dicynene should be used for 1 week only. 